\title{
Evaluating the effective numbers of independent tests and significant $p$-value thresholds in commercial genotyping arrays and public imputation reference datasets
}

\author{
Miao-Xin Li • Juilian M. Y. Yeung • \\ Stacey S. Cherny $\cdot$ Pak C. Sham
}

Received: 23 August 2011/Accepted: 13 November 2011/Published online: 6 December 2011

(C) The Author(s) 2011. This article is published with open access at Springerlink.com

\begin{abstract}
Current genome-wide association studies (GWAS) use commercial genotyping microarrays that can assay over a million single nucleotide polymorphisms (SNPs). The number of SNPs is further boosted by advanced statistical genotype-imputation algorithms and large SNP databases for reference human populations. The testing of a huge number of SNPs needs to be taken into account in the interpretation of statistical significance in such genome-wide studies, but this is complicated by the non-independence of SNPs because of linkage disequilibrium (LD). Several previous groups have proposed the use of the effective number of independent markers $\left(M_{\mathrm{e}}\right)$ for the adjustment of multiple testing, but current methods of calculation for $M_{\mathrm{e}}$ are limited in accuracy or computational speed. Here, we report a more
\end{abstract}

Electronic supplementary material The online version of this article (doi:10.1007/s00439-011-1118-2) contains supplementary material, which is available to authorized users.

M.-X. Li · J. M. Y. Yeung - S. S. Cherny - P. C. Sham Department of Psychiatry, The University of Hong Kong, Pokfulam, Hong Kong

M.-X. Li · P. C. Sham

The Centre for Reproduction, Development and Growth,

The University of Hong Kong, Pokfulam, Hong Kong

M.-X. Li · P. C. Sham

Genome Research Centre, The University of Hong Kong, Pokfulam, Hong Kong

S. S. Cherny · P. C. Sham

State Key Laboratory for Cognitive and Brain Sciences,

The University of Hong Kong, Pokfulam, Hong Kong

P. C. Sham $(\bowtie)$

Department of Psychiatry, LKS Faculty of Medicine,

University of Hong Kong, Pokfulam, Hong Kong

e-mail: pcsham@hkucc.hku.hk robust and fast method to calculate $M_{\mathrm{e}}$. Applying this efficient method [implemented in a free software tool named Genetic type 1 error calculator (GEC)], we systematically examined the $M_{\mathrm{e}}$, and the corresponding $p$-value thresholds required to control the genome-wide type 1 error rate at 0.05 , for 13 Illumina or Affymetrix genotyping arrays, as well as for HapMap Project and 1000 Genomes Project datasets which are widely used in genotype imputation as reference panels. Our results suggested the use of a $p$-value threshold of $\sim 10^{-7}$ as the criterion for genome-wide significance for early commercial genotyping arrays, but slightly more stringent $p$-value thresholds $\sim 5 \times 10^{-8}$ for current or merged commercial genotyping arrays, $\sim 10^{-8}$ for all common SNPs in the 1000 Genomes Project dataset and $\sim 5 \times 10^{-8}$ for the common SNPs only within genes.

\section{Introduction}

Genome-wide association studies (GWAS) can now directly assay up to 2.5 million single nucleotide polymorphisms (SNPs) using high-throughput genotyping arrays (Ragoussis 2009). The number of SNPs is further boosted by statistical genotype-imputation algorithms that make use of large SNP reference datasets such as the HapMap Project and 1000 Genomes Project (Anderson et al. 2008; Howie et al. 2009). The number of SNPs is set to increase further with recent advances in resequencing technology (Metzker 2010). The testing of such huge numbers of SNPs results in a massive multiple-testing burden in statistical analysis.

The Bonferroni correction, which resets the significance threshold from $\alpha$ to $\alpha / M$ in the presence of $M$ independent tests, is probably the most popular method for multipletesting adjustment. However, the Bonferroni correction assumes independence among the tests considered, so that 
it is inherently conservative when considering SNPs in linkage disequilibrium (LD). Adjustment for multiple testing by permutation appropriately takes account of marker dependency and results in a more powerful test (Pahl and Schafer 2010), but is computationally expensive. There have been a number of attempts to extend the conventional Bonferroni procedure to handle correlated tests, by replacing the actual number of markers being tested $(M)$ by a smaller value called the effective number of independent markers $\left(M_{\mathrm{e}}\right)$. This results in a test-wise significance threshold of $\alpha^{\prime}=\alpha / M_{\mathrm{e}}$, which controls the familywise error rate (FWER) at $\alpha$. Conversely, the test-wise error rate $\alpha^{\prime}$ is related to the family-wise error by $\alpha=$ $1-\left(1-\alpha^{\prime}\right)^{M_{\mathrm{e}}} \approx M_{\mathrm{e}} \alpha^{\prime}$ Efforts were made to assess the genome-wide significance thresholds after Bonferroni correction for early GWAS (Dudbridge and Gusnanto 2008; Pe'er et al. 2008). However, it is not known whether these thresholds are still applicable to current or future GWAS in which much more SNPs are assayed.

Several methods have been proposed for estimating $M_{\mathrm{e}}$ from the correlations between the genetic markers. Duggal et al. (2008) suggested the simple method of counting 1 SNP per LD block in addition to all the SNPs outside of blocks. Other proposed methods involved the eigenvalues of the LD measure $r^{2}$ or Pearson correlation matrix of allele counts calculated from all possible pairs of SNPs (Cheverud 2001; Gao et al. 2008; Li and Ji 2005; Nyholt 2004; Galwey 2009). Two of these methods used the variance of the eigenvalues ( $\lambda$ ) to estimate $M_{\mathrm{e}}$ (Cheverud 2001; Nyholt 2004). An important limitation of these variance-based approaches is that they do not result in additive $M_{\mathrm{e}}$ estimates across contiguous sets of SNPs. Li and Ji (2005) suggested summing the eigenvalues, after substituting 1 for the eigenvalues that are greater than 1 . While generally more accurate than the variance-based approaches, this method can be both conservative and liberal in different situations ( $\mathrm{Li}$ and Ji 2005). Gao et al. suggested defining $M_{\mathrm{e}}$ as the number of eigenvalues which can explain $C \%$ of the variation for SNP genotype data. However, it is unclear how $C$ should be set, as overly large or small value of $C$ would result in an FWER that is overly conservative or liberal, respectively (2008). Galwey (2009) proposed a measure of $M_{\mathrm{e}}$ based on an eigenvalue ratio function. Moskvina and Schmidt suggested a formula to approximate $M_{\mathrm{e}}$ based on the conditional probability of a Type 1 error in one marker given the test outcome of a second marker (Moskvina and Schmidt 2008). Several studies have concluded that the available measures of $M_{\mathrm{e}}$ were not sufficiently accurate as a valid substitute for a permutation procedure (Han et al. 2009; Salyakina et al. 2005; Galwey 2009).

Here we propose a new method to more accurately and rapidly estimate the effective number of independent tests, $M_{\mathrm{e}}$, from a given set of SNPs. The ratio of $M_{\mathrm{e}}$ to the actual number of SNPs in a genotyping array is suggested as an index of the tagging efficiency of an array. Extensive simulation studies based on both artificial and real LD patterns were conducted to compare the performance of this method against five alternative approaches. We then systematically investigated the $M_{\mathrm{e}}$ for 13 popular commercial genotyping arrays from Illumina and Affymetrix, as well as for the HapMap Project and 1000 Genomes Project genotype datasets which are widely used as reference panels in genotype imputation. From this, we provide a series of suggested Bonferroni $p$-value thresholds to correct for the multiple-testing burden in different populations, when using these arrays and imputed datasets.

\section{Methods and materials}

Construction of a new measure of the effective number of independent tests

Our method is similar to that of $\mathrm{Li}$ and Ji (2005), except that the used eigenvalues are those of the correlation matrix of association test $p$ values, rather than the correlation matrix of allele counts, between SNPs. In a previous paper (Li et al. 2011), we described a polynomial approximation that allows the correlation matrix of association test $p$ values to be calculated from the correlation matrix of allele counts. If the eigenvalues of the correlation matrix of $M$ association test $p$ values are denoted by $\lambda_{i}$, then the effective number of tests, $M_{\mathrm{e}}$ is estimated to be $M-$ $\sum_{i=1}^{M}\left[I\left(\lambda_{i}>1\right)\left(\lambda_{i}-1\right)\right]$, where $I(x)$ is an indicator function. The second part of this formula estimates the redundant number of tests as a result of marker dependency. The $p$-value threshold to control FWER to $\alpha$, using $M_{\mathrm{e}}$ in a Bonferroni procedure, would then be $\alpha / M_{\mathrm{e}}$. The ratio $R_{\mathrm{e}}=M_{\mathrm{e}} / M$, called "effective ratio" for convenience, measures the extent that the $M$ markers are non-redundant.

A divide-and-conquer algorithm was developed to speed up the calculation of eigenvalues of large correlation matrices. SNPs on a chromosome can be partitioned into multiple loose LD blocks. Within a block, a SNP has strong or moderate LD with at least one other SNP while SNPs in different LD blocks are in weak LD (say, $r^{2}<0.1$ ). The-

oretically, assume a large correlation matrix, $P=$ $\left[\begin{array}{cc}A & 0 \\ 0 & B\end{array}\right]$, has an eigenvalue, $\lambda$, and an associated eigenvector $\left[\begin{array}{l}X \\ Y\end{array}\right]$. According to the definition of eigenvalue, $\left[\begin{array}{cc}A & 0 \\ 0 & B\end{array}\right]\left[\begin{array}{c}X \\ Y\end{array}\right]=\left[\begin{array}{l}A X \\ B Y\end{array}\right]=\left[\begin{array}{l}\lambda X \\ \lambda Y\end{array}\right]$. Therefore, $A X=\lambda X$ and 
$B Y=\lambda Y$. This indicates that matrixes $P, A$ and $B$ share the same eigenvalues and the LD block partition will not change the eigenvalues and thus the resulting $M_{\mathrm{e}}$, provided the blocks are independent. The $M_{\mathrm{e}}$ of whole genome is equal to the summation of the $M_{\mathrm{e}}$ calculated within each LD block. This divide-and-conquer strategy substantially speeds up the analysis by avoiding calculating eigenvalues in a huge matrix with thousands of rows and columns, although, in principle, if blocks cannot be formed the proposed measure of $M_{\mathrm{e}}$ could still be implemented.

\section{Datasets}

\section{Local genotype dataset}

In the simulation study, we used a genotype dataset of 2,514 Chinese subjects typed by the Illumina Human610Quad BeadChip. This sample was originally prepared for several independent disease-gene mapping projects [(Kung et al. 2010) and unpublished data]. After standard quality control procedures of GWAS scan for common variants, 473,931 SNPs were left in the simulation analysis.

\section{HapMap LD dataset}

We downloaded the latest version of pair-wise LD data $\left(r^{2}\right)$ of the 11 HapMap panels (http://hapmap.ncbi.nlm.nih.gov/ downloads/ld_data/latest/, Release 27). For the JPT, CHB, CEU and YRI panels, this release merged SNPs of phases I + II + III and had more SNPs than other 7 panels which entered the HapMap Project at phase III. Therefore, we used the LD data of the 4 panels to derive the $M e$ on the 13 commercial genotyping arrays. The numbers of unique SNPs contained in the $4 \mathrm{LD}$ dataset for JPT, CHB, CEU and YRI panels were 2,509,881, 2,554,939, 2,776,528 and $3,114,362$, respectively. But to provide a reference for GWAS imputation in more populations, we estimated the $M_{\mathrm{e}}$ and corresponding $p$-value thresholds in all of the 11 panels as well.

\section{Genomes Project genotype dataset}

We downloaded genotypes of 1000 Genomes Project (released by August 2010) from the website of $\mathrm{MACH}$ (http://www.sph.umich.edu/csg/abecasis/MACH/download/). In this dataset, there were total 651 individuals separated in three different panels according to ancestry, ASN (Asian, 194), EUR (European, 283), and AFR (African, 174). The numbers of overall SNPs in the three panels are 10,832,281 (ASN), 11,914,767 (EUR), and 17,042,857 (AFR), respectively. However, only around half of the SNPs have the minor allele frequencies over 0.05 . We estimated the $M_{\mathrm{e}}$ among SNPs with minor allele frequencies $\geq 0.05$ because
SNPs with too small minor allele frequency are generally underpowered in GWAS.

Examining the relationship between LD $r^{2}$ and correlation of $p$ values from association tests

Genotype data of two bi-allelic SNPs were simulated for a number of subjects, for a set of LD coefficients, $r$, and allele frequencies, under Hardy-Weinberg equilibrium. For a case-control study, we randomly assigned disease status to generate 3,000 cases and 3,000 controls; for a quantitative trait study the 6,000 subjects were randomly given phenotypic scores sampled from the standard normal distribution $N(0,1)$. That is, we simulated no correlation between trait/disease and genotype. An allelic association test was then performed for each of the two SNPs in the case-control study and the Wald test for parameters in a linear regression model was used to examine association in the quantitative trait study. The procedure was repeated 100,000 times to obtain 100,000 sets of $p$ values, from which the correlation coefficient of the $p$ values of the two SNPs, $\rho$, was calculated. The allele frequencies and the LD coefficients, $r$ (Hill and Robertson 1968), were incremented in steps of 0.05 to generate a series of data points. Repeated simulations using samples of different sizes were also conducted.

The relationship between LD $r^{2}$ and $p$-value correlation coefficients was extrapolated by least-squares fitting using a 6th order polynomial function of the squared pair-wise allelic correlation coefficient, $r^{2}$, in Microsoft Excel 2007. We found that under the null hypothesis $p$-value correlation coefficient, $\rho$, can be accurately approximated by a 6th order polynomial function of the squared pair-wise allelic correlation coefficient $r^{2}$ (coefficient of determination $R^{2}=0.9987$ ) (Supplementary Fig. 1), regardless of allele frequencies, sample size and study design.

Comparison of type 1 error of various measures by simulation and permutation

Given the LD patterns and allele frequencies (see supplementary Table 1), a program based on the HapSim algorithm (Montana 2005) was written to generate genotype data under Hardy-Weinberg equilibrium. We simulated regions with 1 LD block (6 SNPs), 2 LD blocks (10 SNPs), 6 LD blocks (30 SNPs) or 24 LD blocks (120 SNPs). We considered the null model where no SNP had an effect on disease risk. For each scenario, a population of 4,000,000 individuals was generated. A random sample of 3,000 cases and 3,000 controls was drawn from the population, without replacement, and subjected to the different methods of multiple testing. Type 1 error rates under the different 
scenarios were obtained from the proportion of simulated datasets that resulted in at least one significant $p$ value (set at 0.05 ), from 1,000 simulated populations.

We compared the performance of the proposed measure to 4 different estimates of $M_{\mathrm{e}}$ as well as the conventional Bonferroni correction approach. A permutation procedure was also carried out for the comparison. The four previous proposed $M_{\mathrm{e}}$ measures have been described in the "Introduction". In the permutation procedure, the phenotypes of subjects were permuted 1,000 times and the smallest SNP $p$ value in a region at each permutation was chosen to generate the empirical distribution. The resulting permuted $p$ value is equal to the proportion of the generated $p$ values less than the observed one.

\section{Examining type 1 error using a real dataset}

The allelic association test was used to examine association at each SNP with simulated disease status in the real genotype dataset of 2,514 Chinese subjects. The pair-wise LD coefficients, $r^{2}$, were approximated by the square of Pearson correlation of genotypes coded as the number of minor alleles $(0,1$ and 2$)$. The $M_{\mathrm{e}}$ and type 1 error were assessed at five regions containing 100-300 SNPs in different chromosomes sampled randomly. SNPs with minor allele frequency less than 0.05, Hardy-Weinberg equilibrium $p$ value less than 0.001 , or genotype call rate less than $90 \%$ were excluded for this analysis. Type 1 error rates for these regions were obtained from the proportion of simulated phenotype datasets that resulted in significant $p$ values (at FWER 0.05), from 50,000 simulated datasets.

Comparison of type 1 error using multivariate normal distribution (MVN)

On each chromosome, we randomly draw 500 regions with a random number of SNPs ranging from 2 to 100 in the same sample of 2,514 Chinese subjects mentioned above. At each region, $M_{\mathrm{e}}$ was estimated by five different methods and the corresponding $p$-value threshold, $\alpha^{\prime}$, for individual SNPs to control the FWER $(\alpha)$ at 0.05 , was calculated by Bonferroni correction method, $\alpha^{\prime}=\alpha / M_{\mathrm{e}}$. Given $\alpha^{\prime}$, the FWER was calculated by the standard cumulative distribution function of $\operatorname{MVN}\left(0, \sum\right)$ :

$$
\begin{aligned}
1-\int_{A}^{-A} f(x) \mathrm{d} u= & 1-\int_{A}^{-A} \frac{1}{(2 \pi)^{M / 2}\left|\sum\right|^{1 / 2}} \exp \left[-\frac{1}{2}(x-\mu)^{T}\right. \\
& \left.\times \sum^{-1}(x-\mu)\right] \mathrm{d} u
\end{aligned}
$$

where $A=\left[\Phi^{-1}\left(\alpha^{\prime} / 2\right), \ldots, \Phi^{-1}\left(\alpha^{\prime} / 2\right)\right]^{T}$ is a $M$ dimension vector and $\sum$ is the genotypic Pearson correlation coefficient matrix of the $M$ SNPs. We used the R package mvtnorm (http://cran.r-project.org/web/packages/mvtnorm/ index.html) for the numerical integration of the MVN.

Estimating $M_{\mathrm{e}}$ and genome-wide significance thresholds in 13 genotyping arrays

It was noted that some SNPs in the genotyping arrays were not in the HapMap Project. For each array, a pair-wise $r^{2}$ was extracted into a subset from the HapMap LD dataset if both of its SNPs appeared on the genotyping array. The $M_{\mathrm{e}}$ and effective ratio were first estimated for SNPs in the subset. The total $M_{\mathrm{e}}$ of the genotyping array was then approximated by the number of SNPs on the array multiplied by the effective ratio. The $p$-value thresholds for genome-wide significant and highly significant association were equal to 0.05 and 0.001 divided by the total $M_{\mathrm{e}}$ of the genotyping array.

\section{Results}

Comparison of FWER in simulated data

The proposed method was compared to several existing methods as well as permutation testing (the gold standard) by simulation studies. Genotypes were simulated according to artificial LD patterns (Supplementary Table 1), and phenotypes were randomly assigned. As shown in Table 1, the use of the proposed $M_{\mathrm{e}}$ for Bonferroni correction produced FWER values that are generally close to the correct value of 0.05 . As expected, standard Bonferroni correction for $M$ SNPs is conservative. The correction based on Nyholt's $M_{\mathrm{e}}$ was liberal when there is only one LD block, but conservative in the multiple-LD-block scenarios. The $\mathrm{Li}$ and $\mathrm{Ji}$ (2005) method was liberal in all the simulated situations, while the Moskvina and Schmidt (2008) method was slightly conservative in the one-block scenario but became less conservative in the multiple-LD-block scenarios. Generally speaking, the type 1 error rates of Moskvina and Schmidt (2008), Galwey (2009), and the proposed method, along with those obtained via permutation, were comparable in the simulated dataset.

\section{Comparison of FWER in real data}

We further examined the family-wise type I error rates of the modified Bonferroni procedure by $M_{\mathrm{e}}$ in a real GWAS genotype dataset, where the phenotypes were re-assigned at random. The real GWAS data used were on a sample of 2,514 Chinese subjects typed by the Illumina Human610Quad BeadChip. Five regions on different chromosomes were randomly chosen for an empirical validation. 
Table 1 Empirical family-wise type 1 error rates (percentages) of alternative multiple testing corrections in simulated datasets

\begin{tabular}{lllllllr}
\hline \#SNP & $\begin{array}{l}\text { Bonferroni } \\
\text { for \# SNP }\end{array}$ & $\begin{array}{l}\text { Nyholt } \\
(2004)\end{array}$ & Li and Ji (2005) & $\begin{array}{l}\text { Moskvina } \\
\text { and Schmidt (2008) }\end{array}$ & $\begin{array}{l}\text { Galwey } \\
(2009)\end{array}$ & Permutation & Proposed $M_{\mathrm{e}}$ \\
\hline 6 & 2.14 & 6.10 & 5.94 & 4.02 & 4.68 & 4.95 & 4.81 \\
10 & 2.70 & 3.82 & 6.24 & 4.45 & 4.96 & 4.98 & 5.01 \\
30 & 2.84 & 3.11 & 6.59 & 4.67 & 5.22 & 4.91 & 5.28 \\
120 & 2.89 & 3.06 & 6.80 & 4.94 & 5.56 & 4.74 & 5.60 \\
\hline
\end{tabular}

The nominal FWER is 0.05 . We simulated 4 different LD patterns, in which a region may have 1 LD block (including 6 SNPs), 2 LD blocks (including $10 \mathrm{SNPs}$ ), 6 LD blocks (including $30 \mathrm{SNPs}$ ) and $24 \mathrm{LD}$ blocks (including $120 \mathrm{SNPs}$ ), respectively, 40,000 replicates for each scenario. See Supplementary Table 1 for the LD patterns

Table 2 Family-wise error rates and effective number of independent tests in real genotype datasets

\begin{tabular}{|c|c|c|c|c|c|c|c|c|c|c|c|c|c|}
\hline \multirow[t]{2}{*}{ Chromosome } & \multirow[t]{2}{*}{ Position $^{a}$} & \multicolumn{2}{|c|}{ The observed } & \multicolumn{2}{|c|}{ Nyholt (2004) } & \multicolumn{2}{|c|}{$\mathrm{Li}$ and $\mathrm{Ji}(2005)$} & \multicolumn{2}{|c|}{$\begin{array}{l}\text { Moskvina and Schmidt } \\
\text { (2008) }\end{array}$} & \multicolumn{2}{|c|}{ Galwey (2009) } & \multicolumn{2}{|c|}{ Proposed $M_{\mathrm{e}}$} \\
\hline & & \#SNP & Error & \#SNP & Error & \#SNP & Error & \#SNP & Error & \#SNP & Error & \#SNP & Error \\
\hline 1 & $\begin{array}{l}5733711 \\
6877920\end{array}$ & 137 & $2.74 \%$ & 128.8 & $2.97 \%$ & 52.0 & $6.56 \%$ & 63.1 & $5.48 \%$ & 48.7 & $7.04 \%$ & 68.5 & $5.16 \%$ \\
\hline 2 & $\begin{array}{l}105304539 \\
106766191\end{array}$ & 271 & $3.31 \%$ & 264.7 & $3.40 \%$ & 110.0 & $7.45 \%$ & 147.1 & $5.81 \%$ & 100.1 & $8.01 \%$ & 159.3 & $5.43 \%$ \\
\hline 3 & $\begin{array}{l}178265666 \\
179728246\end{array}$ & 186 & $2.97 \%$ & 180.8 & $3.06 \%$ & 85.0 & $6.40 \%$ & 99.3 & $5.54 \%$ & 78.3 & $6.84 \%$ & 106.7 & $5.11 \%$ \\
\hline 6 & $\begin{array}{l}100078150 \\
102098421\end{array}$ & 282 & $2.68 \%$ & 271.7 & $2.78 \%$ & 88.0 & $7.74 \%$ & 126.2 & $5.66 \%$ & 78.6 & $8.51 \%$ & 137.3 & $5.21 \%$ \\
\hline 21 & $\begin{array}{l}30821453 \\
31663481\end{array}$ & 118 & $3.01 \%$ & 113.2 & $3.14 \%$ & 51.0 & $6.86 \%$ & 62.9 & $5.73 \%$ & 48.8 & $7.08 \%$ & 68.1 & $5.26 \%$ \\
\hline
\end{tabular}

${ }^{a}$ The coordinates of NCBI Human Reference Genome Build 36.3 was used to denote the regions. The 5 regions were randomly selected. The Nominal FWER is $0.05 ; 50,000$ simulated replicates were produced for each region

As shown in Table 2, the proposed measure of $M_{\mathrm{e}}$ led to FWERs much closer to the nominal $\alpha=0.05$ for all regions in 50,000 simulated datasets. The simple Bonferroni correction for number of SNPs was conservative, as expected, as was the Bonferroni correction using Nyholt's $M_{\text {e }}$. The methods of Li and Ji (2005) and Galwey (2009) resulted in quite liberal FWERs. The FWERs based on Moskvina and Schmidt (2008) were only slightly more liberal than those based on our new method.

\section{Comparison of FWER via MVN}

For some common tests of association, the vector of test statistics for a single trait over multiple markers asymptotically follows a MVN, or can be transformed to follow a MVN (Lin 2005; Seaman and Muller-Myhsok 2005); the covariance matrix of this MVN can be approximated from the matrix of correlation coefficients between the markers (Moskvina and Schmidt 2008; Han et al. 2009; Seaman and Muller-Myhsok 2005; Conneely and Boehnke 2007). Given a fully characterized MVN, the FWER for any specified SNP-wise error rate can be calculated by multivariate integration. However, this is only feasible for a limited number of SNPs because of the computational burden in calculating the probabilities from a large-dimensional MVN. We randomly drew 500 genomic regions on each of the 22 autosomes and the $\mathrm{X}$ chromosome from the real GWAS dataset mentioned above. The number of markers within each region was random, ranging from 2 to 100 . At each region, the five different methods were used to estimate $M_{\mathrm{e}}$ and to calculate the test-wise $p$-value threshold required to obtain a nominal FWER of 0.05. An estimate of the FWER corresponding to each test-wise $p$-value threshold is then obtained from the MVN. Figure 1 shows a Box plot of the MVN-derived FWER for the different methods over the 11,500 randomly selected regions. The proposed method of estimating $M_{\mathrm{e}}$ appears to give MVN-derived FWERs that agree most closely with the nominal level of 0.05 , with least bias and small variance (Fig. 1). Consistent with the results obtained via simulation and permutation, the Bonferroni correction using Nyholt's $M_{\mathrm{e}}$ was generally conservative; the methods of $\mathrm{Li}$ and Ji (2005) and Galwey (2009) resulted in liberal FWERs, and all three have larger variance across genomic regions. The FWERs based on Moskvina and Schmidt (2008) were slightly more liberal but had comparable variance as the proposed method. 
Fig. 1 Box plot of MVNderived FWERs for five different methods. For each method, the nominal FWER was set to be 0.05 . The bottom and top of each box mark the 25th and 75 th percentile, respectively, and the band in the box denotes the 50th percentile. The lines above and below each box denote the upper and lower 1.5 interquartile range (IQR)

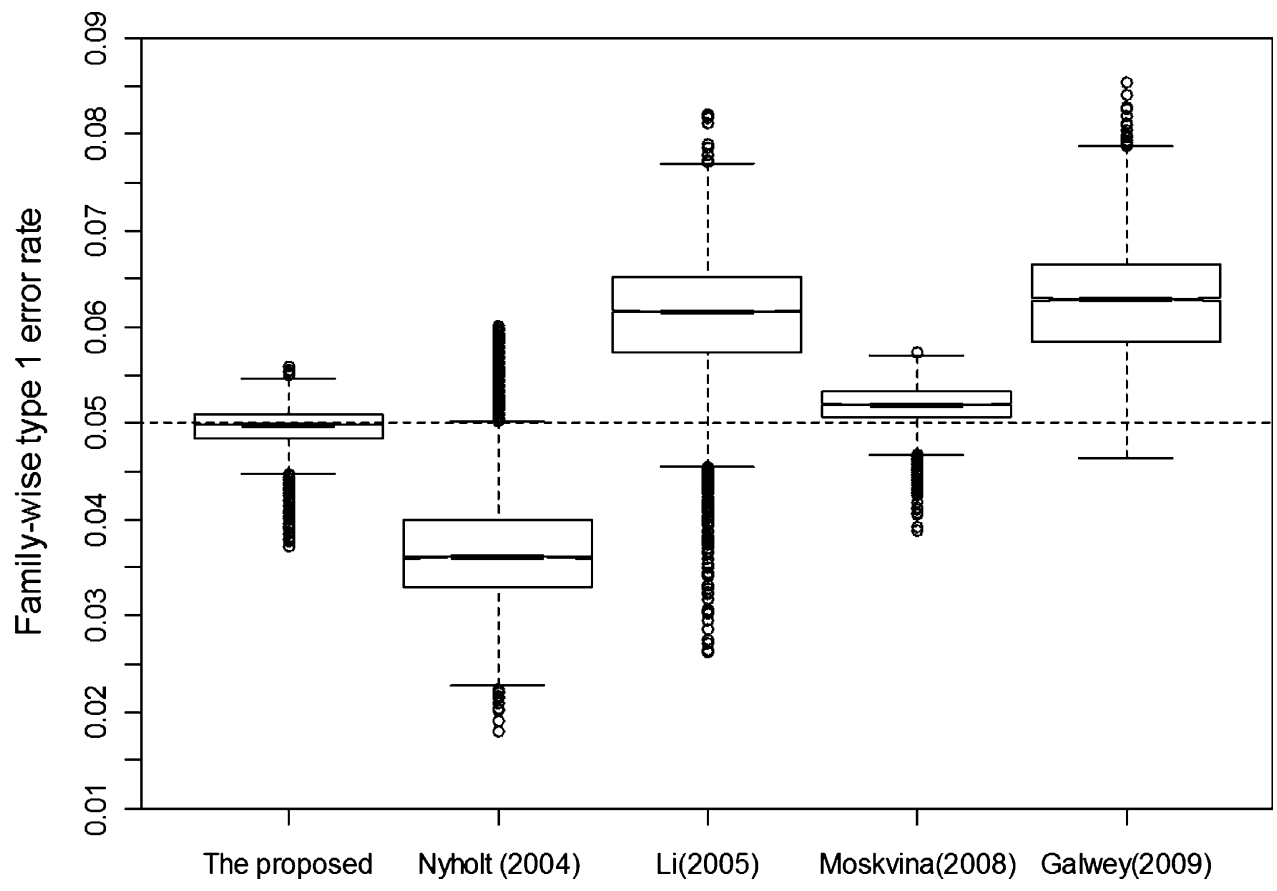

Estimating $M_{\mathrm{e}}$ and genome-wide significance thresholds in 13 genotyping arrays

Applying the proposed method, we systematically estimated $M_{\mathrm{e}}$ for 7 Illumina and 6 Affymetrix genotyping arrays, which have been widely used in GWAS in various populations. The $r^{2}$ values in the HapMap LD dataset (released on April 19, 2009) were used to calculate $p$-value correlation coefficients. Similar to the criteria proposed for genome-wide linkage studies (Lander and Kruglyak 1995), we calculated $p$-value thresholds for two genome-wide significance levels, significant association, and highly significant association, in which the FWER per scan are 0.05 and 0.001 , respectively. Table 3 shows results based on HapMap CEU LD dataset. The thresholds for genome-wide significant association for all genotyping arrays (except for the Illumina HumanOmni2.5) range from $8.21 \times 10^{-8}$ to $1.11 \times 10^{-6}$, which are all slightly less stringent than the widely-adopted one, $5.0 \times 10^{-8}$. An association scan based on the densest Affymetrix array requires a $p$-value threshold of $1.08 \times 10^{-7}$ to declare a significant hit and the corresponding threshold for Illumina HumanHap $1 \mathrm{M}$ is $8.21 \times 10^{-8}$. When combining all of the six Affymetrix arrays $(1,011,854$ unique SNPs in total), the $p$-value threshold for significant association is $1.04 \times 10^{-7}$. The Illumina HumanOmni2.5 seems to have an efficient design for effective SNPs. Its effective ratio is comparable with the Illumina HuamHap $1 \mathrm{M}$ although it has doubled the SNP amount. When all of the seven Illumina arrays were combined, the $p$-value threshold turned out to be $\sim 3.5 \times 10^{-8}$. This amount of markers often happens in GWAS with genotype imputation, particularly in metaanalysis of GWAS. Consistent with observation in Barrett and Cardon (2006), Illumina arrays have larger effective ratio and require more stringent $p$-value thresholds to declare a significant finding than the Affymetrix arrays with similar number of SNPs. Results based on the HapMap CHB, JPT and YRI LD datasets are shown in Supplementary Table 2. Similarly, except for the Illumina HumanOmni2.5, the thresholds for genome-wide significant association using the other available genotyping arrays are all slightly less stringent than the widely adopted threshold, $5.0 \times 10^{-8}$.

Estimating $M_{\mathrm{e}}$ and significance thresholds in datasets of HapMap and 1000 Genomes Project

We then measured the $M_{\mathrm{e}}$ in datasets of HapMap and 1000 Genomes project. As shown in Table 4, although the number of unique SNPs in the HapMap LD dataset is over 2.5 million in the JPT, CHB and CEU panels, the $M_{\mathrm{e}}$ is less than 1 million and the ratio of $M_{\mathrm{e}}$ to the observed number (i.e., the effective ratio) is low, ranging from 0.26 to 0.30 . The $p$-value thresholds for significant association are looser than $5.0 \times 10^{-8}$. The YRI panel has both the largest number of SNPs and effective ratio in the HapMap data, which makes the stringent $p$-value threshold $3.44 \times 10^{-8}$. Supplementary Table 3 shows the estimation results in the other 7 HapMap panels. There are only around 1.5 million SNPs in each panel and the effective ratios range from 0.41 
Table 3 Estimated effective number of SNPs and $p$-value thresholds using the HapMap CEU sample

\begin{tabular}{|c|c|c|c|c|c|c|c|}
\hline & \multirow[t]{2}{*}{ Array Name } & \multicolumn{3}{|l|}{ \#SNP } & \multirow[t]{2}{*}{ Effective ratio } & \multicolumn{2}{|c|}{$p$-value thresholds* } \\
\hline & & In total & $\begin{array}{l}\text { In } \\
\text { HapMap }\end{array}$ & $M_{\mathrm{e}}$ & & $\begin{array}{l}\text { Significant } \\
\text { association }\end{array}$ & $\begin{array}{l}\text { Highly significant } \\
\text { association }\end{array}$ \\
\hline \multirow[t]{7}{*}{ Illumina HumanHap } & Omni2.5 & $2,450,000$ & 969,415 & 544,311 & 0.561 & $3.63 \mathrm{E}-08$ & $7.27 \mathrm{E}-10$ \\
\hline & $1 \mathrm{M}$ & $1,199,187$ & 964,612 & 513,911 & 0.533 & $7.83 \mathrm{E}-08$ & $1.57 \mathrm{E}-09$ \\
\hline & $650 \mathrm{Y}$ & 660,557 & 609,860 & 393,752 & 0.646 & $1.17 \mathrm{E}-07$ & $2.34 \mathrm{E}-09$ \\
\hline & p610-Quad & 598,821 & 561,716 & 374,316 & 0.666 & $1.25 \mathrm{E}-07$ & $2.51 \mathrm{E}-09$ \\
\hline & p550-Duo & 561,122 & 540,047 & 370,501 & 0.686 & $1.30 \mathrm{E}-07$ & $2.60 \mathrm{E}-09$ \\
\hline & CNV370 & 353,188 & 338,660 & 258,305 & 0.763 & $1.86 \mathrm{E}-07$ & $3.71 \mathrm{E}-09$ \\
\hline & 300-Duo & 318,117 & 317,804 & 251,244 & 0.791 & $1.99 \mathrm{E}-07$ & $3.98 \mathrm{E}-09$ \\
\hline \multirow[t]{6}{*}{ Affymetrix array } & Array 6.0 & 934,968 & 783,702 & 388,751 & 0.496 & $1.08 \mathrm{E}-07$ & $2.16 \mathrm{E}-09$ \\
\hline & Array 5.0 & 443,816 & 384,423 & 211,592 & 0.550 & $2.05 \mathrm{E}-07$ & $4.09 \mathrm{E}-09$ \\
\hline & $250 \mathrm{~K}$ Nsp & 262,264 & 227,290 & 141,440 & 0.622 & $3.06 \mathrm{E}-07$ & $6.13 \mathrm{E}-09$ \\
\hline & 250 K Sty & 238,304 & 204,969 & 136,228 & 0.665 & $3.16 \mathrm{E}-07$ & $6.31 \mathrm{E}-09$ \\
\hline & $50 \mathrm{~K}$ Hind 240 & 56,936 & 48,917 & 38,773 & 0.793 & $1.11 \mathrm{E}-06$ & $2.22 \mathrm{E}-08$ \\
\hline & 50 K Xba 240 & 58,625 & 53,624 & 41,219 & 0.769 & $1.11 \mathrm{E}-06$ & $2.22 \mathrm{E}-08$ \\
\hline \multicolumn{2}{|c|}{ Merged illumina arrays } & $3,048,319$ & $1,316,091$ & 617,409 & 0.469 & $3.50 \mathrm{E}-08$ & $6.99 \mathrm{E}-10$ \\
\hline \multicolumn{2}{|c|}{ Merged affymetrix arrays } & $1,011,854$ & 853,412 & 404,187 & 0.474 & $1.04 \mathrm{E}-07$ & $2.09 \mathrm{E}-09$ \\
\hline
\end{tabular}

* $p$-value threshold is equal to the FWER/(Total number of SNPs $\times$ effective ratio)

Table 4 Estimated effective number of SNPs and genome-wide significance thresholds

\begin{tabular}{|c|c|c|c|c|c|c|}
\hline & & \multirow[t]{2}{*}{ \#SNP* } & \multirow[t]{2}{*}{$M_{\mathrm{e}}$} & \multirow[t]{2}{*}{ Effective ratio } & \multicolumn{2}{|l|}{$p$-value thresholds } \\
\hline & & & & & Significant association & Highly significant association \\
\hline \multirow[t]{4}{*}{ HapMap } & JPT panel & $2,509,881$ & $664,279.75$ & 0.26 & $7.53 \mathrm{E}-08$ & $1.51 \mathrm{E}-09$ \\
\hline & CHB panel & $2,554,939$ & $693,418.45$ & 0.27 & $7.21 \mathrm{E}-08$ & $1.44 \mathrm{E}-09$ \\
\hline & CEU panel & $2,776,528$ & $820,888.14$ & 0.30 & $6.09 \mathrm{E}-08$ & $1.22 \mathrm{E}-09$ \\
\hline & YRI panel & $3,114,362$ & $1,452,799.72$ & 0.47 & $3.44 \mathrm{E}-08$ & $6.88 \mathrm{E}-10$ \\
\hline \multirow[t]{3}{*}{1000 Genomes } & ASN (Asian) & $5,367,975$ & $1,442,762.66$ & 0.27 & $3.47 \mathrm{E}-08$ & $6.93 \mathrm{E}-10$ \\
\hline & EUR (European) & $5,730,196$ & $1,634,900.82$ & 0.29 & $3.06 \mathrm{E}-08$ & $6.12 \mathrm{E}-10$ \\
\hline & AFR (African) & $7,961,101$ & $3,091,723.20$ & 0.39 & $1.62 \mathrm{E}-08$ & $3.23 \mathrm{E}-10$ \\
\hline
\end{tabular}

*In the 1000 Genomes dataset, 50.4, 51.9 and 53.2\% SNPs with minor allele frequency below 0.05 were filtered out in the ASN, EUR and AFR panels, respectively

to 0.65 . However, the $p$-value thresholds of significant association are still close to $5.0 \times 10^{-8}$ in four panels (LWK, MKK, ASW and MEX). The CHD panel has the smallest number of SNPs and its $p$-value threshold for significant association is close to $10^{-7}$.

The 1000 Genomes Project samples are divided into three panels according to their population ancestry. The common SNPs with minor allele frequency over 0.05 in the 1000 Genomes Project is over twice as large as the number of SNPs in the HapMap data. The effective ratios in the 1000 Genomes Project datasets of ASN and EUR panel are similar to that in the HapMap dataset of the corresponding populations although the amount of SNPs of the former is much more than that of the latter. The effective ratio in the
1000 Genomes Project AFR panel is smaller than that of HapMap YRI panel. The large $M_{\mathrm{e}}$ in the 1000 Genomes Project datasets entails stringent $p$-value thresholds below $5.0 \times 10^{-8}$ for significant association. These $p$-value thresholds are useful reference for GWAS based on the genotype imputation using genotypes from HapMap and 1000 Genomes as reference sample.

We also estimated potential effective number of SNPs within known genes. Gene regions were defined according to the reference genome coordinates (GRCh37) of its transcripts with $2000 \mathrm{bp}$ extension at both sides. The RefGene dataset was used in this analysis, including 37,322 transcripts of 22,610 genes. Table 5 lists the estimated effective number of SNPs and significance thresholds in the 
Table 5 Estimated effective number of SNPs and significance thresholds in gene regions

\begin{tabular}{llllll}
\hline & \#SNP* & $M_{\mathrm{e}}$ & Effective ratio & \multicolumn{2}{c}{$p$-value thresholds } \\
\cline { 5 - 6 } & & & Significant association & Highly significant association \\
\hline ASN (Asian) & $2,427,784$ & 675845.93 & 0.28 & $7.40 \mathrm{E}-8$ & $1.48 \mathrm{E}-9$ \\
EUR (European) & $2,591,410$ & $765,693.14$ & 0.30 & $6.53 \mathrm{E}-8$ & $1.31 \mathrm{E}-9$ \\
AFR (African) & $3,603,810$ & $1,448,010.91$ & 0.40 & $3.45 \mathrm{E}-8$ & $6.91 \mathrm{E}-10$ \\
\hline
\end{tabular}

*51.0, 52.5 and 53.8\% SNPs with minor allele frequency below 0.05 were excluded in the ASN, EUR and AFR panels, respectively

datasets of 1000 Genomes Project. The effective ratios in the gene regions are slightly higher than those in the whole genome. The $p$-value thresholds for SNPs in gene regions are roughly twice than that for the whole genome SNPs, back to a level close to $5 \times 10^{-8}$.

As expected from known LD patterns of populations worldwide (Frazer et al. 2007), the effective ratio in the Asia population is smaller than that in the European population and the African population has the largest effective ratio in both the HapMap and 1000 Genomes Project datasets. In principle, the effective ratio measures the average LD degree between SNPs in a marker set. A lower effective ratio is resulted from higher degree and/or longerrange of LD between markers. Given the same set of markers, a larger $R_{\mathrm{e}}$ may imply, on average, more miosis and recombination evens per genome happened in a population as a result of longer population history. Therefore, the largest effective ratio in the African population also indirectly supports the longest history of this population and is consistent with the 'Out of Africa' event hypothesis (Tishkoff et al. 1996; Reich et al. 2001). Correspondingly, the required $p$-value threshold for significant association in the African population is the more stringent those in the Asian and European population.

A software tool to estimate $M_{\mathrm{e}}$ and type I error

We have implemented the proposed measure of effective number of independent tests and the improved Bonferroni correction procedure in a software tool named genetic type 1 error calculator (GEC, http://statgenpro.psychiatry.hku.hk/ gec/). Users can input actual genotype data [in either the conventional linkage pedigree format or PLINK binary format (Purcell et al. 2007)] or the HapMap LD data into GEC to quickly calculate $M_{\mathrm{e}}$ of the whole genome or at some specified genomic regions. Table 6 lists the running time GEC took to estimate $M_{\mathrm{e}}$ by the proposed method on 6 Illumina genotyping arrays using HapMap CEU LD data. If a set of SNP $p$ values for genetic association tests is input, GEC will straightforwardly report the significant SNPs according to the improved Bonferroni correction procedure. GEC has both user-friendly command line and web-based graphic online interface.
Table 6 The running time GEC need to scan various genotyping arrays

\begin{tabular}{lll}
\hline Array name & \#SNP & Running time $(\min )^{\mathrm{a}}$ \\
\hline 1 M-Duo & $1,199,187$ & $\sim 7.8$ \\
$650 \mathrm{Y}$ & 660,557 & $\sim 3.1$ \\
p610-Quad & 598,821 & $\sim 2.7$ \\
p550-Duo & 561,122 & $\sim 2.5$ \\
CNV370 & 353,188 & $\sim 1$ \\
300-Duo & 318,117 & $\sim 0.9$ \\
\hline
\end{tabular}

The configuration of the computer doing the test is Intel(R) Xeon(R) X5670 @ 2.97 GHz, and Ubuntu 11.04 64bit; One GB maximal memory was set for GEC

a The time needed to read HapMap LD data was also included

\section{Discussion}

In the present study, we proposed a more robust measure of the effective number of independent tests, $M_{\mathrm{e}}$, to control FWER for genetic association studies. Compared with previous methods (Gao et al. 2008; Li and Ji 2005; Moskvina and Schmidt 2008; Nyholt 2004; Galwey 2009), our measure is more robust to variable LD patterns in real datasets. Moreover, the new measure is additive across multiple distinct LD blocks. Capitalizing on this property, we developed a divideand-conquer algorithm to handle large datasets, which can substantially relieve the computational burden when scanning millions of SNPs by avoiding calculating eigenvalues of the massive correlation matrix. We have demonstrated that this new method yields correct type I error rates and behaves similarly to the gold standard of permutation.

Pe'er et al. (2008) estimated the multiple testing burden in GWAS through simulation studies using data on the HapMap ENCODE regions to emulate an infinitely dense map, analogous to the Lander and Kruglyak approach for linkage analysis (Lander and Kruglyak 1995), and arrived at the commonly accepted genome-wide significance threshold of $5 \times 10^{-8}$. Similarly, by subsampling genotypes at increasing density and extrapolating to infinite density, Dudbridge and Gusnanto, 2008) estimated the genome-wide significance threshold to be about $7.2 \times 10^{-8}$. We noted that for 12 arrays widely used by previous GWAS, the recommended threshold for a 
genome-wide error rate of $0.05,5.0 \times 10^{-8}$, is conservative. For some studies, using arrays of $\sim 500,000$ or 600,000 SNPs, a $p$-value threshold of $\sim 10^{-7}$ can be safely adopted without inflation of type I error. However, for GWAS using one of the latest Illumina arrays, HumanOmni2.5, a threshold as stringent as $5 \times 10^{-8}$ or even slightly smaller is needed. This is also true for GWAS employing imputed common SNPs based on HapMap data. For GWAS with several million imputed SNPs from the 1000 Genomes Project dataset, a slightly more stringent $p$ value threshold $\left(10^{-8}\right)$ is necessary. However, if one only examines the imputed SNPs within known genes, the threshold $5.0 \times 10^{-8}$ can be used.

As previously noted (Pe'er et al. 2006; Hao et al. 2008), GWAS employing Affymetrix arrays allow use of a less stringent $p$-value threshold than those employing Illumina with similar amount of markers because Affymetrix randomly selected their SNPs while Illumina used a tagging approach in designing their arrays. Consistent with previous reports (Barrett and Cardon 2006; Pe'er et al. 2008), the multiple-testing burden for a sample from Japanese and Chinese populations is less heavy than that for a sample from Caucasian and African populations. Hence, the exact thresholds of individual GWAS slightly vary across genotyping platforms and sampling populations. We provided a user friendly tool, GEC, to quickly calculate exact genome-wide thresholds.

The effective ratio, $R_{\mathrm{e}}$, we proposed can aid in marker selection for genetic association study design as well. Undoubtedly, a design with $R_{\mathrm{e}}$ close to 0 is not cost-efficient because this implies that most typed markers will be redundant and little independent information will be obtained. The larger the $R_{\mathrm{e}}$ is, the more independent genotype information the SNP marker set will have. Meanwhile, it should be noted that solely using $R_{\mathrm{e}}$ to evaluate a design may not be sufficient. It is possible that only one of two imperfectly correlated markers is in strong LD with an untyped disease susceptibility locus (DSL). Exclusion of one marker can definitely increase the $R_{\mathrm{e}}$ but can result in a loss of statistical power if the only marker in strong LD with a DSL is removed. Therefore, there is not a perfect relationship between $R_{\mathrm{e}}$ and statistical power.

In the present paper, we did not investigate the performance of the proposed method in an imputed GWAS dataset. The imputation quality, which is often related to imputation quality thresholds employed to clean the dataset, imputation algorithms and even matching degree between the study sample and reference panels, may affect the estimation of $M_{\mathrm{e}}$ in an imputed genotype dataset. If the quality of imputed genotypes are poor and the pair-wise LD between SNPs calculated by the imputed genotypes is largely different from that by actual genotypes, the estimation of $M_{\mathrm{e}}$ using the imputed dataset will be not reliable.
On the other hand, if the imputation quality is good and the pair-wise LD between SNPs calculated according to the imputed genotypes is very similar to that by actual genotypes, the proposed method can be safely applied to estimate the $M_{\mathrm{e}}$ in the imputed GWAS datasets. In the present study, we estimated the $M_{\mathrm{e}}$ in the public datasets (including the HapMap Project panels and 1000 Genomes Project panels) which are widely used as reference panels for GWAS imputation. The $M_{\mathrm{e}}$ and $p$-value thresholds in these reference panels can be regarded as reference boundaries for the imputed GWAS datasets. In practice, one can employ our tool, GEC, to quickly estimate the $M_{\mathrm{e}}$ in an specific imputed GWAS datasets given the imputation quality is good.

Acknowledgments We thank HapMap Project for the LD data and 1000 Genomes Project for the genotype data used in this project. This work was funded HKU 7672/06 M, the Small Project Funding HKU 201007176166, the European Community's Seventh Framework Programme under grant agreement No. HEALTH-F2-2010-241909 and The University of Hong Kong Strategic Research Theme on Genomics.

Open Access This article is distributed under the terms of the Creative Commons Attribution Noncommercial License which permits any noncommercial use, distribution, and reproduction in any medium, provided the original author(s) and source are credited.

\section{References}

Anderson CA, Pettersson FH, Barrett JC, Zhuang JJ, Ragoussis J, Cardon LR, Morris AP (2008) Evaluating the effects of imputation on the power, coverage, and cost efficiency of genome-wide SNP platforms. Am J Hum Genet 83(1):112-119. doi:10.1016/j.ajhg.2008.06.008

Barrett JC, Cardon LR (2006) Evaluating coverage of genome-wide association studies. Nat Genet 38(6):659-662. doi:10.1038/ ng1801

Cheverud JM (2001) A simple correction for multiple comparisons in interval mapping genome scans. Heredity $87(\mathrm{Pt} 1): 52-58$ (901[pii])

Conneely KN, Boehnke M (2007) So many correlated tests, so little time! Rapid adjustment of P values for multiple correlated tests. Am J Hum Genet 81 (6). doi:10.1086/522036

Dudbridge F, Gusnanto A (2008) Estimation of significance thresholds for genomewide association scans. Genet Epidemiol 32(3):227-234. doi:10.1002/gepi.20297

Duggal P, Gillanders EM, Holmes TN, Bailey-Wilson JE (2008) Establishing an adjusted p-value threshold to control the familywide type 1 error in genome wide association studies. BMC Genomics 9:516. doi:10.1186/1471-2164-9-516

Frazer KA, Ballinger DG, Cox DR, Hinds DA et al (2007) A second generation human haplotype map of over 3.1 million SNPs. Nature 449(7164):851-861. doi:10.1038/nature06258

Galwey NW (2009) A new measure of the effective number of tests, a practical tool for comparing families of non-independent significance tests. Genet Epidemiol 33(7):559-568. doi:10.1002/ gepi. 20408 
Gao X, Starmer J, Martin ER (2008) A multiple testing correction method for genetic association studies using correlated single nucleotide polymorphisms. Genet Epidemiol 32(4):361-369. doi:10.1002/gepi.20310

Han B, Kang HM, Eskin E (2009) Rapid and accurate multiple testing correction and power estimation for millions of correlated markers. PLoS Genet 5(4):e1000456. doi:10.1371/journal.pgen. 1000456

Hao K, Schadt EE, Storey JD (2008) Calibrating the performance of SNP arrays for whole-genome association studies. PLoS Genet 4(6):e1000109. doi:10.1371/journal.pgen.1000109

Hill WG, Robertson A (1968) Linkage disequilibrium in finite populations. Theor Appl Genet 38(4):226-231

Howie BN, Donnelly P, Marchini J (2009) A flexible and accurate genotype imputation method for the next generation of genomewide association studies. PLoS Genet 5(6):e1000529. doi: 10.1371/journal.pgen.1000529

Kung AW, Xiao SM, Cherny S, Li GH, Gao Y, Tso G, Lau KS, Luk KD, Liu JM, Cui B, Zhang MJ, Zhang ZL, He JW, Yue H, Xia WB, Luo LM, He SL, Kiel DP, Karasik D, Hsu YH, Cupples LA, Demissie S, Styrkarsdottir U, Halldorsson BV, Sigurdsson G, Thorsteinsdottir U, Stefansson K, Richards JB, Zhai G, Soranzo N, Valdes A, Spector TD, Sham PC (2010) Association of JAG1 with bone mineral density and osteoporotic fractures: a genomewide association study and follow-up replication studies. Am J Hum Genet 86(2):229-239. doi:10.1016/j.ajhg.2009.12.014

Lander E, Kruglyak L (1995) Genetic dissection of complex traits: guidelines for interpreting and reporting linkage results. Nat Genet 11(3):241-247. doi:10.1038/ng1195-241

Li J, Ji L (2005) Adjusting multiple testing in multilocus analyses using the eigenvalues of a correlation matrix. Heredity 95(3):221-227. doi:10.1038/sj.hdy.6800717

Li MX, Gui HS, Kwan JS, Sham PC (2011) GATES: a rapid and powerful gene-based association test using extended simes procedure. Am J Hum Genet 88(3):283-293. doi:10.1016/j.ajhg. 2011.01.019

Lin DY (2005) An efficient Monte Carlo approach to assessing statistical significance in genomic studies. Bioinformatics 21(6):781-787. doi:10.1093/bioinformatics/bti053

Metzker ML (2010) Sequencing technologies-the next generation. Nat Rev Genet 11(1):31-46. doi:10.1038/nrg2626

Montana G (2005) HapSim: a simulation tool for generating haplotype data with pre-specified allele frequencies and LD coefficients. Bioinformatics 21(23):4309-4311. doi:10.1093/bio informatics/bti689
Moskvina V, Schmidt KM (2008) On multiple-testing correction in genome-wide association studies. Genet Epidemiol 32(6):567573. doi:10.1002/gepi.20331

Nyholt DR (2004) A simple correction for multiple testing for singlenucleotide polymorphisms in linkage disequilibrium with each other. Am J Hum Genet 74(4):765-769. doi:10.1086/383251

Pahl R, Schafer H (2010) PERMORY: an LD-exploiting permutation test algorithm for powerful genome-wide association testing. Bioinformatics 26(17):2093-2100. doi:10.1093/bioinformatics/ btq399

Pe'er I, de Bakker PI, Maller J, Yelensky R, Altshuler D, Daly MJ (2006) Evaluating and improving power in whole-genome association studies using fixed marker sets. Nat Genet 38(6):663-667. doi:10.1038/ng1816

Pe'er I, Yelensky R, Altshuler D, Daly MJ (2008) Estimation of the multiple testing burden for genomewide association studies of nearly all common variants. Genet Epidemiol 32(4):381-385. doi:10.1002/gepi.20303

Purcell S, Neale B, Todd-Brown K, Thomas L, Ferreira MA, Bender D, Maller J, Sklar P, de Bakker PI, Daly MJ, Sham PC (2007) PLINK: a tool set for whole-genome association and populationbased linkage analyses. Am J Hum Genet 81(3):559-575. doi: $10.1086 / 519795$

Ragoussis J (2009) Genotyping technologies for genetic research. Annu Rev Genomics Hum Genet 10:117-133. doi:10.1146/ annurev-genom-082908-150116

Reich DE, Cargill M, Bolk S, Ireland J, Sabeti PC, Richter DJ, Lavery T, Kouyoumjian R, Farhadian SF, Ward R, Lander ES (2001) Linkage disequilibrium in the human genome. Nature 411(6834):199-204. doi:10.1038/35075590

Salyakina D, Seaman SR, Browning BL, Dudbridge F, MullerMyhsok B (2005) Evaluation of Nyholt's procedure for multiple testing correction. Hum Hered 60 (1):19-25; discussion 61-12. doi: $10.1159 / 000087540$

Seaman SR, Muller-Myhsok B (2005) Rapid simulation of P values for product methods and multiple-testing adjustment in association studies. Am J Hum Genet 76(3):399-408. doi:10.1086/ 428140

Tishkoff SA, Dietzsch E, Speed W, Pakstis AJ, Kidd JR, Cheung K, Bonne-Tamir B, Santachiara-Benerecetti AS, Moral P, Krings M (1996) Global patterns of linkage disequilibrium at the CD4 locus and modern human origins. Science 271(5254):1380-1387 